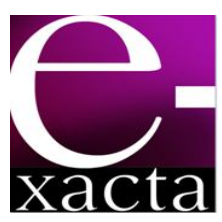

ISSN: 1984-3151

\section{MODELAGEM, SIMULAÇÃO E OTIMIZAÇÃO DA DINÂMICA OPERACIONAL DO PROCESSO DE ATENDIMENTO DE UM PEQUENO POSTO DE SAÚDE}

\section{MODELING, SIMULATION AND OPTIMIZATION OF THE OPERATIONAL DYNAMIC OF THE ATTENDING PROCESS OF A SMALL HEALTH POST}

\author{
José Airton A. dos Santos'; Tiago C. Dal Sotto; Wesley Schroeder ${ }^{3}$ \\ 1 Doutor em Engenharia Elétrica. UFSC, 1999. Professor do \\ Curso de Engenharia de Produção da Universidade \\ Tecnológica Federal do Paraná. Medianeira - PR. \\ airton@utfpr.edu.br \\ 2 Graduando do Curso de Engenharia de Produção da \\ Universidade Tecnológica Federal do Paraná. Medianeira - \\ PR. tiagodalsotto@hotmail.com \\ 3 Graduando do Curso de Engenharia de Produção da \\ Universidade Tecnológica Federal do Paraná. Medianeira - \\ PR. wesleyschroeder@hotmail.com
}

Recebido em: 19/03/2013 - Aprovado em: 20/04/2013 - Disponibilizado em: 11/06/2013

RESUMO: Este trabalho tem como objetivo analisar a dinâmica operacional do processo de atendimento de um pequeno posto de saúde localizado na região oeste paranaense. Objetiva-se também através da associação dos conceitos de simulação e otimização maximizar o número de agendamento de consultas para o posto de saúde, dentro do limite de acomodação da sala de espera. A simulação e a otimização foram executadas utilizando o pacote de simulação Arena®, que inclui o software de otimização Optquest. A metodologia utilizada é a de modelagem através de simulação computacional, de caráter quantitativo e é caracterizada como participativa. $A$ aplicação destas técnicas em conjunto resultaram na otimização do número de agendamento de consultas médicas do posto de saúde.

Palavras-Chave: Arena®. Posto de saúde. Otimização. Simulação.

This work aims to analyze the attending process operational dynamics of a small health post located in Paraná West Region. Another objective is connect the concepts of simulation and optimization to maximize the number of scheduling appointments for the health post, within the limits of accommodation of the waiting room. The simulation and optimization were performed using the Arena $\AA$ simulation package, which includes the OptQuest optimization software. The methodology used was the modeling through computer simulation of quantitative character and it is characterized as participative. The application of these techniques all together resulted in the optimization of the number of medical appointment scheduling of the health post.

KEYWORDS: Arena @ ${ }^{\circledR}$. Health post. Optimization. Simulation.

\section{INTRODUÇÃo}

No Brasil, um posto de saúde, é o lugar onde encontram-se médicos para atender à população de um bairro ou de uma determinada região.

$O$ atendimento é gratuito e destina-se exclusivamente à prevenção. Os casos mais graves e/ou urgências, emergências, devem se encaminhar diretamente a um pronto-socorro, onde há recursos adequados para tais atendimentos. No posto de saúde, o paciente agenda as consultas.

O mistério da saúde defende a ideia de que a saúde é um direito de todos os cidadãos (BRASIL, 2013). 
Porém, observa-se que ainda existe deficiência no sistema de saúde atual, onde há falta de profissionais especializados, além de uma estrutura física apropriada nos postos de saúde para uma assistência adequada à saúde da população. Além disso, o baixo poder aquisitivo das famílias menos privilegiadas contribui para o agravamento das doenças em geral, pois a demanda de pacientes é grande e a fila nas salas de espera dos postos de saúde ainda permanece excessiva.

O fenômeno de formação de filas já é rotineiro na vida atual, ocorre em diversas aplicações, como uma peça esperando para ser lixada ou polida (na indústria), um avião esperando para decolar (em um aeroporto), um programa de computador esperando para ser executado, e, é claro, uma fila de seres humanos esperando serviço (BANKS, 1998).

As filas se formam em decorrência do aumento dos consumidores e da incapacidade do sistema em atender a essa demanda. Assim, através de técnicas de simulação, busca-se encontrar um ponto de equilíbrio que satisfaça os clientes e seja viável economicamente para o provedor do serviço (ARENALES et al., 2007).

Segundo Banks (1998), simulação é uma técnica de solução de um problema pela análise do modelo que descreve o comportamento de um sistema usando um computador digital. A simulação de um modelo permite entender a dinâmica de um sistema, assim como, analisar e prever o efeito de mudanças que se introduzem nele. É uma representação próxima da realidade, e será tanto mais real quanto mais características significativas do sistema seja capaz de representar. Por outro lado, o modelo deve ser simples, de forma que não se torne demasiado complexo para se construir, mas, ao mesmo tempo, deve ser o mais fiel possível ao sistema real (CHWIF; MEDINA, 2007).
Inicialmente, os sistemas de simulação foram desenvolvidos sobre linguagens de programação de propósito geral, tais como: Fortran, Basic, Pascal, etc. Porém, isso exigia um grande esforço para a construção de modelos, além de profissionais com conhecimentos profundos de programação de computadores. Diante dessa dificuldade é que começaram a surgir linguagens de programação, dedicadas à simulação, que superassem essa barreira. É o caso, por exemplo, das linguagens Gpss, Siman, Slam, Simscript, etc. Tais linguagens eram, na verdade, bibliotecas formadas por conjuntos de macro comandos das linguagens de propósito gerais. Alguns dos simuladores da geração seguinte foram desenvolvidos sobre a plataforma dessas linguagens. Como exemplo tem-se 0 software Arena®, implementado na linguagem Siman (KELTON; SADOWSKI, 1998).

A otimização é o processo de tentar diferentes combinações de valores para variáveis que podem ser controladas, buscando aquela que provê a saída mais desejada de um modelo de simulação. Existem atualmente vários softwares que permitem a realização da otimização a partir de uma simulação. Dentre eles, pode-se citar: AutoStat, OptQuest, OPTIMIZ, SimRunner® e WITNESS Optimizier, que fazem parte de pacotes comerciais onde também está incluso o módulo de simulação (RYAN; HEAVEY, 2006).

Diante do exposto, este trabalho tem como objetivo analisar, através de técnicas de simulação e de otimização, a dinâmica operacional do processo de atendimento de um pequeno posto de saúde, localizado na região oeste paranaense.

\section{MATERIAIS E MÉtodos}

A metodologia utilizada neste estudo pode ser classificada quanto ao tipo de pesquisa, a população 
amostral, a coleta e a análise dos dados. Desta forma serão apresentados os passos seguidos para a realização do trabalho.

A classificação, quanto ao tipo de pesquisa, pode ser subdividida de acordo com a natureza, sendo esta aplicada, quanto aos objetivos sendo descritiva, quanto à forma de abordar o problema, pode ser considerada quantitativa, pois os dados obtidos (cronometrados) no sistema real foram, em seguida, tratados estatisticamente.

Como estratégia de pesquisa, foi utilizado o estudo de caso que, conforme Yin (2001), é ideal em situações organizacionais reais em que o pesquisador não tem controle dos fenômenos.

\subsection{O Posto de Saúde}

No posto de saúde, ocorrem atendimentos diários a moradores (do bairro ou adjacências), no período matutino, feito por uma recepcionista, uma enfermeira e dois médicos. Os pacientes chegam com hora marcada e são atendidos no balcão da recepção, logo após são encaminhados para uma sala de espera, onde aguardam para medições de peso e pressão. Ao término, retornam para a sala à espera da consulta com o médico.

Delimitado o sistema a ser simulado e definido o objetivo do trabalho partiu-se então para a construção do modelo conceitual. A etapa de criação do modelo conceitual é o aspecto mais importante de um estudo de simulação (MIRANDA et al., 2012).

O ponto de partida deste trabalho foi o modelo conceitual, que forneceu informações ao modelo computacional. A representação do modelo conceitual, na forma de Fluxograma, é apresentada na Figura 1.

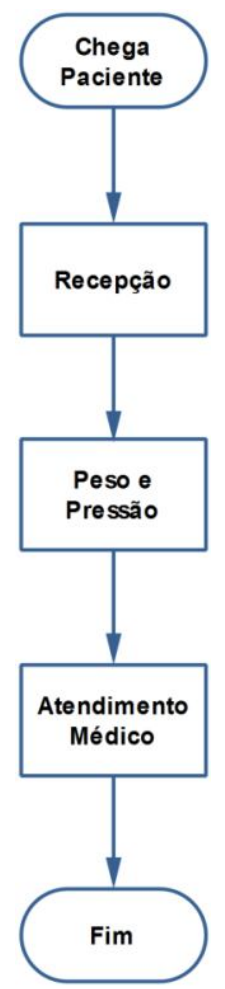

Figura 1 - Fluxograma do sistema

$\mathrm{Na}$ Figura 2 apresenta-se o layout do sistema em estudo.

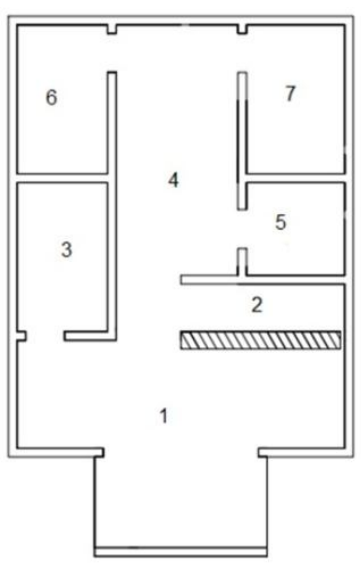

1 - Entrada

2 - Balcão

3- Banheiro

4 - Sala de Espera

5 - Sala de medição de peso e pressão

6 e 7 - Consultórios médicos

Figura 2 - Layout do posto de saúde

\subsection{O SOFTWARE AREna}

O Arena $\AA^{\circledR}$ é um ambiente gráfico integrado de simulação, que contém inúmeros recursos para modelagem, animação, análise estatística e análise de 
resultados. A plataforma de simulação Arena ${ }^{\circledR}$, segundo Keyton e Sadowski (1998), possui as seguintes ferramentas:

i. Analisador de dados de entrada (Input Analyzer);

ii. Analisador de resultados (Output Analyzer);

iii. Analisador de processos (Process Analyzer);

iv. Otimizador (Optquest).

Este software é composto por um conjunto de blocos (ou módulos) utilizados para se descrever uma aplicação real e que funcionam como comandos de uma linguagem de programação. Os elementos básicos da modelagem em Arena® são as entidades que representam as pessoas, objetos, transações, etc, que se movem ao longo do sistema; as estações de trabalho que demonstram onde será realizado algum serviço ou transformação, e por fim, o fluxo que representa os caminhos que a entidade irá percorrer ao longo de estações (FERNANDES et al., 2006).

Para Fu (2002), na interação entre simulação e otimização, a última deve ser vista como uma ferramenta complementar à simulação. Neste processo, a otimização fornece as variáveis de uma possível solução (inputs) à simulação; esta, após todo o processo de simulação, fornece respostas (outputs) para a situação proposta, que retornam à otimização. A otimização gera novas variáveis, utilizando técnicas específicas, que serão novamente testadas pela simulação. Este ciclo é repetido até sua parada, definida de acordo com o método de otimização utilizado.

A combinação entre simulação e otimização faz com que as respostas sejam mais eficientes, possibilitando uma melhor tomada de decisão.

Para validação do modelo conceitual utilizou-se a técnica de validação face a face (SARGENT, 1998). Através de reuniões entre os analistas e os funcionários do posto de saúde foi possível confirmar a validação do modelo, após pequenas correções no decorrer do desenvolvimento.

No planejamento da coleta de dados, concluiu-se que seria necessário determinar as seguintes variáveis: tempo entre chegadas dos pacientes (TEP); tempo de atendimento na recepção (TAR), tempo de atendimento na medição do peso e da pressão (TPP) e tempo de atendimentos nos consultórios médicos (TAM). Observa-se que os dados foram coletados no mês de setembro de 2012.

Os dados foram analisados com a ferramenta Input analyzer (analisador de dados de entrada) do software Arena ${ }^{\circledR}$. Segundo Prado (2010) esta ferramenta permite analisar dados reais do funcionamento do processo e escolher a melhor distribuição estatística que se aplica a eles.

A simulação da dinâmica operacional do posto de saúde foi realizada com o software Arena $\AA^{\circledR}$, e os resultados analisados nas ferramentas Output Analyzer e Process Analyzer.

\subsection{NÚMERO DE REPLICAÇõES}

Segundo Freitas Filho (2008), de uma maneira geral, a coleta de dados para a composição de uma amostra a partir da simulação de um modelo pode ser realizada de duas formas:

1. Fazer uso das observações individuais dentro de cada replicação. Por exemplo, pode-se simular o processo e utilizar o tempo que cada paciente esperou na fila da medição de peso e pressão para realizar uma estimativa do tempo médio de espera na fila. Neste caso, o tamanho da amostra será igual à quantidade de pacientes que passaram pela fila ao longo do período simulado.

2. Realizar $n$ simulações (replicações), onde cada replicação gera um elemento para a amostra. Uma vez que se está lidando com um sistema terminal 
no qual as condições iniciais e o período de simulação são fixos, a melhor maneira de garantir que os valores da amostra sejam estatisticamente independentes é obtê-los a partir de replicações independentes.

Neste trabalho, o número de replicações $\left(n^{*}\right)$ foi obtido através da Eq. (1):

onde:

$$
\mathrm{n}^{*}=\mathrm{n} \times\left(\frac{\mathrm{h}}{\mathrm{h}^{*}}\right)^{2}
$$

n: número de replicações já realizadas;

h: semi-intervalo de confiança já obtido; e

$\mathrm{h}^{*}$ : semi-intervalo de confiança desejado.

\subsection{VALIDAÇÃo do MOdelo}

Segundo Sargent (1998), o processo de validação do sistema é uma etapa fundamental, pois é ele que determina se o modelo proposto detém precisão suficiente para representar o sistema real. Desta forma se o modelo não é uma aproximação do sistema real, todas as conclusões derivadas deste estarão susceptíveis a erros e poderão resultar em decisões incorretas. Sendo assim a validação pode e deve ser feita para todos os modelos, independentemente do sistema (LAW; KELTON, 2000).

$\mathrm{Na}$ execução do procedimento de validação, para o sistema em estudo, utilizou-se o erro médio estimado (Eq. 2) (MONTGOMERY, 2005):

$$
\mathrm{SE}=\sqrt{\frac{(\mathrm{SR}-\mathrm{MD})^{2}}{\mathrm{GLR}}}
$$

onde:

SE - erro médio estimado;

SR - valor obtido a partir do sistema real;

MD - média dos valores gerados pelo modelo; e

GLR - grau de liberdade considerando o número de replicações do modelo.

\subsection{TAMANHO DA AMOSTRA}

O tamanho de cada uma das quatro amostras, cronometradas neste trabalho, foi obtido para um nível de confiança de 95\%, através da Eq. (3) (MARROCO 2003):

$$
\mathrm{n}_{\mathrm{A}}=\left(\frac{\angle \alpha_{\mathrm{z}} \times \mathrm{s}}{\mathrm{E}}\right)^{2}
$$

onde:

$\mathrm{n}_{\mathrm{A}}$ : número de indivíduos da amostra;

$Z_{\alpha 2}$ : valor crítico que corresponde ao grau de confiança desejado;

S: desvio padrão;

E: erro máximo estimado.

\section{Resultados e Discussão}

Foi realizada uma avaliação descritiva completa dos dados coletados no posto de saúde no software Statistica ${ }^{\circledR}$ versão 10. A Tabela 1 apresenta, como exemplo, os dados coletados: Tempo entre Chegadas dos Pacientes (TEP).

Tabela 1 - Análise dos dados - TEP

\begin{tabular}{c|c}
\hline Parâmetro analisado & TEP \\
\hline Pontos & $414 \mathrm{~s}$ \\
Média & $82,78633 \mathrm{~s}$ \\
Mediana & $54,3437 \mathrm{~s}$ \\
Mínimo & $1,22623 \mathrm{~s}$ \\
Máximo & $587,095 \mathrm{~s}$ \\
1 Quartil $\left(\mathrm{Q}^{1}\right)$ & $21,557 \mathrm{~s}$ \\
3 Quartil (Q $\left.{ }^{3}\right)$ & $116,844 \mathrm{~s}$ \\
Desvio Padrão & $85,20718 \mathrm{~s}$ \\
Coeficiente de Variação & $103 \%$ \\
\hline
\end{tabular}

Segundo Pimentel (2000), nos experimentos de campo, se o coeficiente de variação for inferior a 10\% 
tem-se um coeficiente de variação baixo, de 10 a $20 \%$ médio, de 20 a $30 \%$ alto e acima de $30 \%$ muito alto.

Após a análise exploratória, realizou-se à análise de correlação entre os dados, ou seja, verificou se há dependência entre os valores das amostras. Na Figura 3 é apresentado, como exemplo, o gráfico de dispersão do Tempo entre Chegadas dos Pacientes no posto. Nessa figura pode-se comprovar que não há correlação entre as observações da amostra.

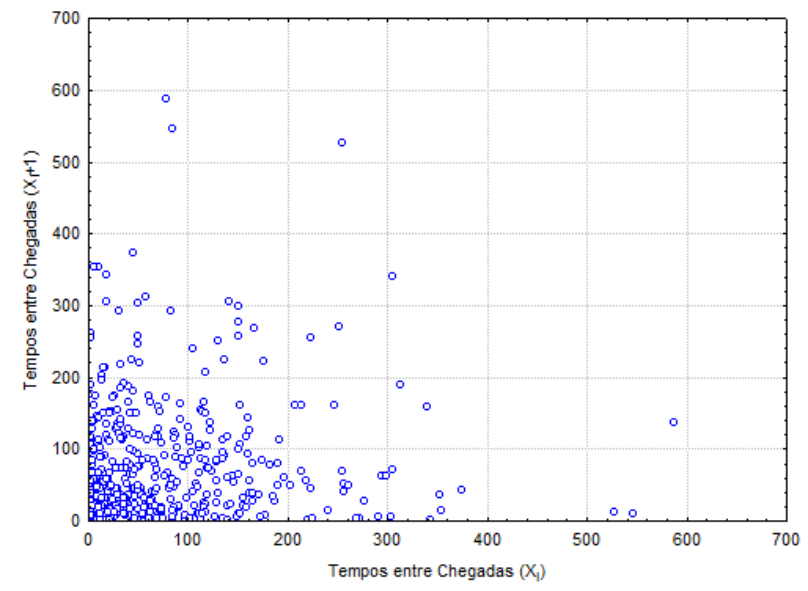

Figura 3 - Gráfico de dispersão - TEP

\subsection{TRATAMENTO DE DADOS}

Inicialmente, os dados foram plotados em forma de boxplot (Figura 4) para uma análise preliminar do comportamento das observações. A seguir, aplicou-se a técnica de identificação de outliers (valores fora da normalidade) apresentada na Tabela 2 (MARROCO, 2003). As razões mais comuns para o surgimento desses valores são os erros na coleta de dados ou eventos raros e inesperados. Os outliers considerados como extremos só foram descartados, das amostras, depois de uma análise criteriosa de suas causas. Os valores julgados como possíveis de ocorrer foram mantidos nas amostras.

e-xacta, Belo Horizonte, v. 6, n. 1, p. 101-109. (2013). Editora UniBH. Disponível em: www.unibh.br/revistas/exacta/

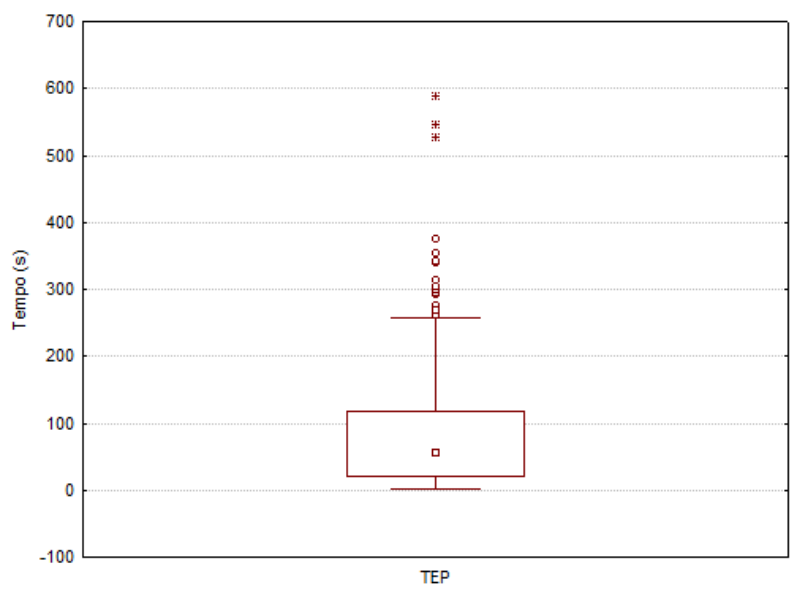

Figura 4 - Boxplot - TEP

Tabela 2 - Identificação de outliers

\begin{tabular}{l}
\multicolumn{1}{c}{ Outliers } \\
\hline$A=Q^{3}-Q^{1}$ \\
Valor $<Q^{1}-1,5 A$ - Outlier Moderado \\
Valor $>Q^{3}+1,5 A$ - Outlier Moderado \\
Valor $<Q^{1}-3,0 A$ - Outlier Extremo \\
Valor $>Q^{3}+3,0 A$ - Outlier Extremo \\
\hline
\end{tabular}

Onde $Q^{1}$ e $Q^{3}$ são, respectivamente, os valores do primeiro e terceiro quartis, assim a amplitude entre inter-quartil " $A$ " é calculada pela diferença: $A=Q^{3}-Q^{1}$.

Após a análise dos dados cronometrados no sistema, através de técnicas estatísticas (MARIN; TOMI, 2010), - passo seguinte foi determinar as curvas de distribuição teórica de probabilidades que melhor representem o comportamento estocástico do sistema em estudo, através da ferramenta Input Analyzer do Arena $\AA^{\circledR}$. Como os $p$-values dos testes de aderência: teste Chi Square e do teste Kolmogorov-Smirnof são maiores que o nível de significância adotado $(0,1)$ (CHWIF; MEDINA, 2007), concluiu-se que as distribuições, apresentadas na Tabela 3 , são as expressões que melhor se adaptaram aos dados coletados no sistema. 
Tabela 3 - Distribuição de probabilidades

\begin{tabular}{cccc}
\hline Item & Distribuição & Chi Square & Kolmogorov-Smirnov \\
\hline TEP & $1+$ GAMM $(92.9,0.852)$ & p-value $=0.208$ & $p$-value $=0.745$ \\
TAR & 4+EXPO $(73)$ & p-value $=0.922$ & $p$-value $=0.598$ \\
TPP & $114+\operatorname{EXPO}(88)$ & $p$-value $=0.609$ & $p$-value $=0.601$ \\
TAM & UNIF $(300,900)$ & $p$-value $=0.506$ & -value $=0.929$ \\
\hline
\end{tabular}

\subsection{VALIDAÇÃO DO MODELO IMPLEMENTADO}

Inicialmente, a validação, do modelo computacional, foi realizada por meio da técnica face a face, onde o modelo foi executado para os funcionários do posto de saúde, que o consideraram correto. $\mathrm{Na}$ sequência realizou-se uma comparação (Tabela 4) entre o número máximo de pacientes atendidos obtido do sistema real com a média gerada pelo modelo para a variável Número de Pacientes Atendidos (NPA). Nesta tabela apresenta-se, também, o erro médio estimado (SE, em decimal).

Tabela 4 - Dados do sistema real e do modelo

\begin{tabular}{c|c|c}
\hline \multicolumn{3}{c}{ Número de Pacientes Atendidos NCA } \\
\hline Sistema Real & Modelo Computacional & SE \\
\hline 40 & $39,5 \pm 3$ & 0,08 \\
\hline
\end{tabular}

Através da análise dos resultados da Tabela 4 podese concluir que o modelo computacional apresenta uma boa aproximação, em relação ao número máximo de pacientes atendidos durante o período analisado (4 horas), com o sistema real.

\subsection{OTIMIZAÇÃo}

Identificou-se, através de observação in loco, que o "gargalo" do sistema em estudo, está na capacidade da sala de espera (máximo de 10 pacientes). Em dias de agendamento máximo, 40 consultas, a sala fica superlotada. Portanto, decidiu-se encontrar, por meio do software optquest, o número máximo de pacientes que podem ser atendidos no posto de saúde, com a condição de não ultrapassar a capacidade máxima da sala de espera.

O problema foi definido da seguinte forma:

\section{Função Objetivo}

Maximizar o número de pacientes atendidos no posto de saúde.

\section{Restricão}

(Fila Peso/Pressão + Fila Consultórios) $\leq 10$ pacientes

\section{Variáveis de controle}

Número de Agendamentos - Mínimo:30 - Máximo: 40.

O Optquest realizou 100 simulações, com 38 replicações cada, para encontrar a melhor solução segundo as opções e os parâmetros definidos. O melhor valor obtido para função objetivo, número máximo de pacientes atendidos no posto, é apresentado na Figura 5.

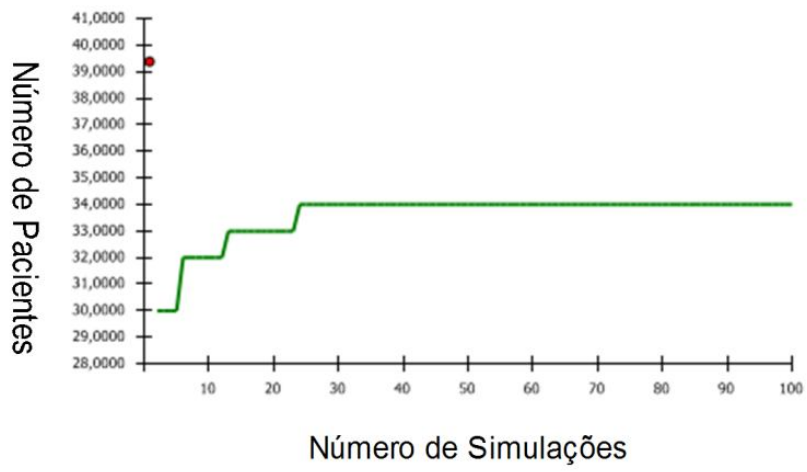

Figura 5 - Número máximo de pacientes 
Por meio do gráfico (Figura 5), obtido através da ferramenta de otimização Optquest do software Arena ${ }^{\circledR}$, pode-se observar que para oferecer um serviço de qualidade aos pacientes, no posto de saúde, deve-se ofertar um agendamento de no máximo 34 consultas.

\subsection{SimULAÇÃo}

Para a realização da análise do sistema em estudo, são propostos dois cenários com o objetivo de observar a resposta do sistema a partir de alterações no número máximo de consultas. Os indicadores de desempenho utilizados para a análise são: a porcentagem de utilização dos funcionários (enfermeira e médicos) e o tamanho da fila da sala de espera (Fila Peso/Pressão + Fila Consultórios).

- Cenário 1 (Cenário atual): Número Máximo de 40 consultas.

- Cenário 2: (Otimização) Número Máximo de 34 consultas.

A Tabela 5 apresenta os resultados obtidos da simulação do sistema, para os dois cenários.

Tabela 5: Resultados de simulação dos cenários 1 e 2

\begin{tabular}{c|c|c|c|c}
\hline \multirow{2}{*}{ Cenários } & Fila & Fila & \multicolumn{2}{|c}{ Utilização } \\
\cline { 4 - 5 } & Peso/Pressão & Consultórios & Enfermeira & Médicos \\
\hline 1 & 11 & 9 & $80 \%$ & $97 \%$ \\
\hline 2 & 4,7 & 4 & $55 \%$ & $80 \%$ \\
\hline
\end{tabular}

Por meio dos resultados obtidos de simulação constatou-se que o cenário 1 é impraticável devido à superlotação da sala (20 pacientes) e ao alto grau de utilização dos médicos (97\%). Portanto, considerando os resultados apresentados na Tabela 3 , pode-se afirmar que o cenário 2 é o melhor cenário simulado neste trabalho.

\section{CONCLUSÕES}

Neste trabalho apresentou-se a metodologia utilizada na implementação do modelo computacional usado para simular a dinâmica operacional do sistema de atendimento de um pequeno posto de saúde na região oeste paranaense.

Umas das vantagens de se utilizar técnicas de simulação e otimização em conjunto é a possibilidade de descobrir com antecedência, o melhor resultado para um determinado contexto e se este é realmente possível.

De acordo com os resultados das análises procedidas concluiu-se que:

- o número máximo de agendamento de consultas adotado atualmente no posto de saúde é impraticável, devido ao alto grau de utilização dos médicos e da superlotação da sala de espera;

- para melhorar o sistema de atendimento do posto de saúde, deve-se agendar no máximo 34 consultas (17 consultas por médico).

A aplicação da simulação computacional gerou um conhecimento adicional acerca do processo de atendimento para todos os envolvidos e possibilitou a identificação de oportunidades de melhorar o sistema de atendimento do posto de saúde. 


\section{REFERÊNCIAS}

ARENALES, M. et al. Pesquisa Operacional. 6. ed. Rio de Janeiro: Elsevier, 2007.

BANKS, J. Handbook of simulation: principles, methodology, advances, applications, and Practice. New York: John Wiley \& Sons, 1998.

BRASIL Ministério da Saúde. Manual do Humaniza: SUS. Ministério da Saúde, 2003a. Disponível em portal.saude.gov.br/portal/saude/cidadao/area.cfm?id_ area=1342. Acesso em: 24 de maio de 2013.

CHWIF, L.; MEDINA, A. C. Modelagem e simulação de eventos discretos, teoria \& aplicações. São Paulo: Brazilian Books, 2007.

FERNANDES, C. A.; SILVA, C. S.; PEREIRA, J. O. YAMAGUCHI, M. M. Simulação da Dinâmica Operacional de uma Linha Industrial de Abate de Suínos. Ciência e Tecnologia de Alimentos, v. 26, p. 166-70. 2006.

FREITAS FILHO, P. J. Introdução à Modelagem e Simulação de Sistemas com Aplicações em Arena. Florianópolis: Visual Books, 2008.

FU, M. C. Optimization for Simulation: Theory vs. Practice. Journal on Computing, v. 14, n 3, 2002.

LAW, A. M.; KELTON, W. D. Simulation modeling and analysis. New York: McGraw- Hill, 2000.

KELTON, W. D.; SADOWSKI, R. Simulation with arena. New York: McGraw-Hill, 1998.
MARIN, T.; TOMI, G. F. C. Modelagem de dados de entrada para simulação estocástica del lavra. Revista Escola de Minas, v. 60, p. 559-62. 2010.

MIRANDA, R. C. et al. Análise de uma unidade de processamento de roupas de um hospital através da simulação a eventos discretos. Produto \& Produção, v. 13, n. 3, p. 07-24, 2012.

MONTGOMERY, D. C. Design and Analysis of Experiments. New York: Wiley, 2005.

MARROCO, J. Análise estatística de dados - com utilização do SPSS. Lisboa: Sílabo, 2003.

PRADO, D. Usando o ARENA em simulação. Nova Lima: INDG - Tecnologia e Serviços LTDA, 2010.

PIMENTEL, F. G. Curso de estatística experimental. Piracicaba: Degaspari, Piracicaba, 2000.

RYAN, J.; HEAVEY, C. Process modeling for simulation. Computers in Industry. v. 57, p. 437-50. 2006.

SARGENT, R. G. Verification and validation of simulation models. In: WINTER THE SIMULATION CONFERENCE, 1998, Proceedings... Washington: WSC, p. 20-8, 1998.

YIN, R. K. Estudo de caso: planejamento e métodos. Porto Alegre: Bookman, 2001. 